\title{
Multi-Variant LRP-Polynomials and Homotopy of Polynomial Equations Systems
}

\author{
ALEKSANDR POLIAKOV \\ Polytechnical Institute \\ Sevastopol State University \\ 33, Universitetskaya Street, Sevastopol, 299053 \\ RUSSIAN FEDERATION \\ a.m.poljakov@sevsu.ru https://orcid.org/0000-0002-2940-8945
}

NARINA KOLESOVA

Institute of Information Technology and Systems and Control Engineering

33, Universitetskaya Street, Sevastopol, 299053

RUSSIAN FEDERATION

makolesova@sevsu.ru

PAVEL BUGAYOV

Marine Institute

Sevastopol State University

33, Universitetskaya Street, Sevastopol, 299053

RUSSIAN FEDERATION

pabugayov@sevsu.ru

\begin{abstract}
Particular case of nonlinear equations are polynomial equations, solving algorithms of which are justified and investigated in the most detail. However, a general approach to solving such equations and their systems that could be considered universal for solving most practical problems has not yet been developed. This is an incentive to search for new algorithms, adapted, at least, to solve typical applied problems. This paper is devoted to the development the method of Laguerre's type for solving polynomial equations systems with real coefficients. It is shown that this method is close in form to the method of homotopy, which effective, for example, in solving optimization problems of nonconvex functions. Its efficiency and advantages in comparison with the known methods are demonstrated on examples of the study of mathematical models of real objects and processes.
\end{abstract}

Key-Words: - Polynomial equation, polynomial equations system with real coefficients, numerical method, Newton method, method of homotopy, Laguerre's type method.

Received: September 26, 2019. Revised: March 27, 2020. Accepted: April 18, 2020. Published: April 30, 2020.

\section{Introduction}

The problem of roots finding of nonlinear equations and their systems has attracted the attention of researchers for several centuries, due to which many methods for solving it are available in the scientific literature. Despite this, it remains one of the most important problems of computational mathematics, due to the need to solve a large number of applied problems, which models are represented by nonlinear equations and their systems.

To systems of polynomial equations are come down mathematical models of many problems of kinematics and dynamics of multilink mechanisms with finite number degrees of freedom, optimization problems, analysis of cyclic molecules, combustion processes and chemical equilibrium, studies of the generalized eigenvalue problem, economic processes, and many others. It is known that in most cases numerical methods and, most often, Newton's method or its modifications are used to study such models. In recent years, evolutionary and probabilistic methods have received significant development. However, as we know, the use of these methods is not always effective.

Consider the following problem. Let $G$ be an open subset of space $\mathbb{R}^{n}: G \subset \mathbb{R}^{n}$, on which is given a sufficiently smooth mapping $\mathcal{F}: G \rightarrow \mathbb{R}^{n}$. It is required to find a solution to the equation $\mathcal{F}(X)=0$ with respect to the unknown vector $\mathcal{X} \in G$. 
Even if we assume that there is a compression mapping $x_{(i+1) k}=\Phi\left(X_{i k}\right)$ constructed by the given equation $\mathcal{F}(\mathcal{X})=0$, which converges to a fixed point $X_{k}^{*}$, situations are possible in which to find numerical solution of the equation $\mathcal{F}(X)=0$ using classical numerical methods is not possible. However, in such cases, it is possible to find a solution of problem by a homotopy method, in which the original vector equation $\mathcal{F}(\mathcal{X})=0$ is replaced by one of easily solved equations, which, in turn, after several iterations goes to the original equation [1-6]. The main difficulty in this case lies precisely in the selection of such easily solvable equation.

Let $\mathcal{H}: G \times[0,1] \rightarrow \mathbb{R}^{n}-$ smooth mapping satisfying the condition:

$$
\mathcal{H}(X, 1)=\mathcal{F}(X), \quad \chi \in G,
$$

and the equation

$$
\mathcal{H}(X, 0)=0
$$

has a solution $X_{0 k} \in G$, which can be easily found.

The operator $\mathcal{H}$ allows to carry out homotopy of the mapping $\mathcal{H}(\circ, 0)$ to the mappi $\mathcal{H}(\circ, 1) \equiv \mathcal{F}$, or, in another way, a smooth transition from easily solved equation (2) to equation (1). Therefore, homotopy is nothing more than a continuously differentiable function

$$
\mathcal{H}(X(q), q)=0, q \in[0,1] .
$$

Thus, the iterative process implemented in accordance with the homotopy method begins with solving the problem, which at the initial stage has a simple (or known) solution, which, in turn, is "deformed" by continuously changing the parameter $\mathrm{q}$ until the solution of original problem will be obtained.

If we assume that the mapping $\mathcal{H}(\circ, q): G \rightarrow \mathbb{R}^{n}$ has an unique zero $\zeta(q), \forall q \in[0,1]$, that is,

$$
\mathcal{H}(\zeta(q), q)=0,
$$

then $\zeta(1)=X_{k}^{*}$ is a desired solution to the original equation $\mathcal{F}(X)=0$.

In cases when defined in this way mapping $\zeta:[0,1] \rightarrow G$ : is smooth, then after differentiation (3), we obtain

$$
\left.\frac{\partial \mathcal{H}}{\partial \mathcal{X}^{T}}\right|_{\chi=\zeta(q)} \zeta^{\prime}(q)+\left.\frac{\partial \mathcal{H}}{\partial q}\right|_{\chi=\zeta(q)}=0,
$$

when $\frac{\partial \mathcal{H}}{\partial X^{T}}=\left(\frac{\partial \mathcal{H}_{i}(\mathcal{X}, q)}{\partial x_{j}}\right)_{1 \leq i, j \leq n}$ is the Jacobi matrix of the mapping $\mathcal{H}(\mathcal{X}, q)$ calculated relatively to the vector $X$ at $X=\zeta(q)$.
If the matrix $\frac{\partial \mathcal{H}}{\partial x^{T}}$ is non-degenerate, then equation (4) is solvable with respect to the $\zeta^{\prime}(q)=$ $\frac{d \zeta(q)}{d q}$ and can be written in the following form:

$$
\zeta^{\prime}(q)=-\left.\left(\left.\frac{\partial \mathcal{H}}{\partial \mathcal{X}^{T}}\right|_{\chi=\zeta(q)}\right)^{-1} \frac{\partial \mathcal{H}}{\partial q}\right|_{\chi=\zeta(q)} .
$$

In fact, this relation can be used to replace the equation $\mathcal{F}(\mathcal{X})=0$ by the Cauchy problem for the system of ordinary differential equations with dummy time $q \in[0,1]$ and the initial condition $x_{0 k} \in G$, which is the zero of the mapping $\mathcal{H}(\circ, 0)$, which is homotopic to the original maping $\mathcal{F}$. This approach is called the Davydenko homotopy method [7] can be implemented in different ways.

The main aim of this work is to substantiate an effective homotopy method for solving a system of polynomial equations with real coefficients, realized through iterations of the Laguerre's type.

\section{The Method Justification}

\subsection{LRP-Polynomials and Laguerre's Type Homotopy}

Consider the polynomial equation

$$
p_{n}(x)=\sum_{j=0}^{n} a_{n-j} x^{j}=0
$$

where $p_{n}(x)$ is a polynomial from a $\operatorname{ring} R[x], x \in$ $\mathbb{R}, a_{j} \in \mathbb{R}, j=0,1, \ldots, n, n \in \mathbb{N}$, as well as the family of polynomials generated by it, depending on the parameter $v \neq 1$ :

$$
p_{n}(v, x)=\sum_{j=0}^{n} a_{n-j}[1-(n-j) v] x^{j} .
$$

The properties of polynomials from family (6), which were called polynomials with a linear real parameter (LRP-polynomials), are described in [8]. We transform the right side of the expression (6) and present it in the following form:

$$
\begin{aligned}
& \quad p_{n}(v, x)=\sum_{j=0}^{n} a_{n-j} x^{j}-v \sum_{j=0}^{n}(n- \\
& \text { j) } a_{n-j} x^{j}=\quad p_{n}(x)-v g_{n-1}(x) .
\end{aligned}
$$

In [8] was proved following lemma:

Lemma 1 . If for any real number $v \neq 1$ the equality $p_{n}(v, x)=p_{n}(x)$ is true, then there exists $x=x_{r} \in$ $\mathbb{R}$ for which $g_{n-1}\left(x_{r}\right)=0$.

Indeed, if we randomly choose two real numbers $v_{k}$ and $v_{m}$, then, taking into account (7), we will have $p_{n}\left(v_{k}, x\right)=p_{n}\left(v_{m}, x\right)$ and, therefore:

$$
\left(v_{k}-v_{m}\right) g_{n-1}(x)=0 .
$$

For $v_{k}=v_{m}$, equation (8) holds for all $\boldsymbol{x} \in \mathbb{R}^{m}$. Otherwise, if $x=x_{r} \in \mathbb{R}^{m}$ is the root of (8), then 
$g_{n-1}\left(x_{r}\right)=0$. That is, the graphs of all LRPfunctions $f_{v}(x)=p_{n}(v, x), v \neq 1$ intersect at points whose abscissas are real roots $x=x_{r(k)}, k \leq n-1$ of the equation $g_{n-1}\left(x_{r}\right)=0$. For $x_{r}=0$, i.e. in cases where the equation $g_{n-1}\left(x_{r}\right)=0$ has no real roots, the graphs of LRP-functions do not intersect. Thus, we can assume that the family of LRPpolynomials (6) defines a continuous homotopy of the polynomial function $p_{n}(x)$ [7].

In [9], it was shown that homotopy

$$
x=\varphi(x)=x \frac{g_{n-1}(x)}{g_{n-1}(x)-p_{n}(x)},
$$

being constructed based on LRP-polynomials from family (6), it is guaranteed to have a single fixed point if it is a compression mapping. Therefore, the iterative process

$$
\begin{gathered}
x_{k}=\frac{x_{k-1}}{1-v_{k-1}}=x_{k-1} \frac{1}{1-\frac{p_{n}\left(x_{k-1}\right)}{g_{n-1}\left(x_{k-1}\right)}}= \\
x_{k-1} \frac{g_{n-1}\left(x_{k-1}\right)}{g_{n-1}\left(x_{k-1}\right)-p_{n}\left(x_{k-1}\right)},
\end{gathered}
$$

converges and $\lim _{x \rightarrow \infty} x_{k}=x_{r}$ - is a fixed point of the mapping (10).

The iterative formula (10) also can be represented as follows:

$$
x_{k+1}=x_{k}-\frac{p_{n}\left(x_{k}\right)}{p_{n}^{\prime}\left(x_{k}\right)-(n-1) \frac{p_{n}\left(x_{k}\right)}{x_{k}}-},
$$

whence it follows that it differs from the classical Newton's iterative formula by the presence of the second term in the denominator.

In addition, formula (11) corresponds to one of the iterative formulas of the Laguerre's type

$$
x_{k+1}=x_{k}-\frac{\alpha+1}{\alpha s_{1}\left(x_{k}\right) \pm\left[(\alpha+1) s_{2}\left(x_{k}\right)-\alpha s_{1}\left(x_{k}\right)^{2}\right]^{\frac{1}{2}}},
$$

which, in the case of $\alpha=\frac{1}{n-1}$, has the form [10]:

$$
x_{k+1}=x_{k}-
$$

$\frac{n f\left(x_{k}\right)}{f^{\prime}\left(x_{k}\right) \pm\left[(n-1)^{2} f^{\prime}\left(x_{k}\right)^{2}-n(n-1) f\left(x_{k}\right) f^{\prime \prime}\left(x_{k}\right)\right]^{\frac{1}{2}}}$.

Back in 1968, O.N. Tikhonov showed that formula (11) is a special case of (12) and, therefore, can be assigned to one of the Laguerre's type families [11].

It should be noted that the authors of this paper obtained iterative formula (11) based on an algorithm that significantly differs from the Tikhonov form, namely, the new algorithm uses the real parameter $v$, which defines the continuous homotopy $\mathcal{H}(v, x)$ of the polynomial $p_{n}(x)$ :

$$
\mathcal{H}(v, x)=p_{v}(v, x)=p_{n}(x)-v g_{n-1}(x) .
$$

During the iterative process, this parameter decreases thereby bringing $\mathcal{H}(v, x)$ to $p_{n}(x)$. In fact, the parameter $v$ at each iteration allows us to estimate the degree of deformation $\mathcal{H}(v, x)$ from a given value of $x$. That is, if we assume that $\exists x_{k} \in$ $\mathbb{R}: \mathcal{H}\left(v_{k}, x_{k}\right)=p_{n}\left(x_{k}\right)-v_{k} g_{n-1}\left(x_{k}\right)=0$, then the degree of deformation $v_{k}$, defined by the formula

$$
v_{k}=\frac{p_{n}\left(x_{k}\right)}{g_{n-1}\left(x_{k}\right)},
$$

can be used to approach $\mathcal{H}(v, x)$ with a smaller deviation from $p_{n}(x)$. To do this, it is necessary to calculate $\quad x_{k+1}=\frac{x_{k}}{1-v_{k}}, \quad$ which gives $v_{k+1}=$ $\frac{p_{n}\left(x_{k+1}\right)}{g_{n-1}\left(x_{k+1}\right)}$. The new value of the parameter $v$ calculated in this way, hypothetically allows us to bring $\mathcal{H}(v, x)$ closer to $p_{n}(x)$.

One of the advantages of the described algorithm is that it can be generalized to systems of polynomial equations [12]. When including a subsection you must use, for its heading, small letters, 12pt, left justified, bold, Times New Roman as here.

\subsection{Multi-Variant LRP-Polynomials and Laguerre's Type Homotopy of Polynomial Systems}

Consider a multi-variant polynomial

$$
\begin{gathered}
P_{S}^{i}\left(x_{1}, \ldots, x_{m}\right)= \\
\sum_{k_{1}=0}^{n_{1}} \ldots \sum_{k_{m}=0}^{n_{2}} a_{n_{1}-k_{1}, \ldots, n_{m}-k_{m}} x_{1}^{k_{1}} \cdot \ldots \cdot x_{m}^{k_{m}},
\end{gathered}
$$

from a ring $R\left[x_{1}, \ldots, x_{m}\right]$ of polynomials with $m$ variables over the field $\mathbb{R}: a_{n_{1}-k_{1}, \ldots, n_{m}-k_{m}} \in \mathbb{R}$; $n_{1}, \ldots, n_{m} \in \mathbb{N}$, where $S=\operatorname{deg}\left(P_{S}^{i}\right)$ is a degree of the $P_{S}^{i}$, to which corresponds the following polynomial equation

$$
P_{S}^{i}\left(x_{1}, \ldots, x_{m}\right)=0 .
$$

If an arbitrary $m$-dimensional vector $R^{0}=$ $\left(r_{1}^{0}, \ldots, r_{m}^{0}\right)^{T}, r_{1}^{0}, \ldots, r_{m}^{0} \in \mathbb{R}$, is not a solution of the equation (15), then suppose that the solution is an $m$-dimensional vector

$$
R^{1}=\left(\frac{r_{1}^{0}}{1-v_{1}^{0}}, \ldots, \frac{r_{m}^{0}}{1-v_{m}^{0}}\right)^{T},
$$

where $\quad\left\{v_{j}^{0} \in \mathbb{R}: v_{j}^{0} \neq 1\right\}, j=1, \ldots, m \quad$ are components of error vector

$$
E^{0}=\left(v_{1}^{0}, \ldots, v_{m}^{0}\right)^{T} .
$$


Then $P_{1}^{i}=P_{S}^{i}\left(\frac{r_{1}^{0}}{1-v_{1}^{0}}, \ldots, \frac{r_{m}^{0}}{1-v_{m}^{0}}\right) \leq \delta \approx 0, \quad$ that allows you to record

$$
\begin{gathered}
\mathcal{H}(E, X)=P_{S}^{i}\left(v_{1}^{0}, \ldots, v_{m}^{0}, r_{1}^{0}, \ldots, r_{m}^{0}\right)= \\
P_{S}^{i}\left(r_{1}^{0}, \ldots, r_{m}^{0}\right)-G_{S-1}^{i} E^{0},
\end{gathered}
$$

where $X=\left(x_{1}, \ldots, x_{m}\right)^{T}$ is a column vector of variables of polynomial equation (15); $G_{S-1}^{i}=$ $\left(g_{1}^{i}, \ldots, g_{m}^{i}\right)$ is a row vector whose components are values of polynomial $g_{j}^{i}, j=1, \ldots, m$ degrees of $S_{j}=\operatorname{deg}\left(g_{j}^{i}\right) \leq S-1$ in the point $R^{0}$.

Polynomials $g_{j}^{i}$ are obtained in the natural way as coefficients for $v_{j}^{0}$ in the polynomial equation (15) after substituting the components of the vector (16) into it, if we neglect the products $v_{j}^{0}$. $v_{k}^{0}, \forall j, k=1, \ldots, m$ and take into account that $v_{j}^{0}, v_{k}^{0} \neq 1$.

The described procedure makes it possible to construct an algorithm of the iterative process for solving polynomial equations systems similar to (10).

To do this, we consider a finite system of equations of the form (15):

$$
\left\{\begin{array}{c}
P_{S_{1}}^{1}\left(x_{1}, \ldots, x_{m}\right)=0 \\
\vdots \\
P_{S_{m}}^{m}\left(x_{1}, \ldots, x_{m}\right)=0
\end{array}\right.
$$

where $S_{1}, \ldots, S_{m}$ are degrees of polynomial equations with numbers $1, \ldots, \mathrm{m}$, respectively.

Applying the method of obtaining expression (18), we come (in the general case) to a system of $m$ heterogeneous linear algebraic equations

$$
\left\{\begin{array}{c}
P_{S_{1}}^{1}\left(r_{1}^{0}, \ldots, r_{m}^{0}\right)-G_{S_{1}-1}^{1}\left(r_{1}^{0}, \ldots, r_{m}^{0}\right) E^{0}=0 \\
\vdots \\
P_{S_{m}}^{m}\left(r_{1}^{0}, \ldots, r_{m}^{0}\right)-G_{S_{m}-1}^{m}\left(r_{1}^{0}, \ldots, r_{m}^{0}\right) E^{0}=0
\end{array}\right.
$$

In matrix form the system (20) has the form

$$
\mathcal{P}_{S}^{0}-\mathcal{G}_{S-1}^{0} E^{0}=0
$$

where $S=\max _{j}\left(S_{j}\right) ; \mathcal{P}_{S}^{0}=\left.\left(P_{S_{1}}^{1}, \ldots, P_{S_{m}}^{m}\right)^{T}\right|_{R^{0}}$; $\mathcal{G}_{S-1}^{0}=\left.\left(G_{S_{1}-1}^{1}, \ldots, G_{S_{m}-1}^{m}\right)^{T}\right|_{R^{0}}$, and $E^{0}$ is the vector determined in accordance with (17).

Assuming that the matrix $\mathcal{G}_{S-1}^{0}$ is nondegenerate and $\operatorname{det} \mathcal{G}_{S-1}^{0} \neq 0$, we multiply (21) from the left by $\left(\mathcal{G}_{S-1}^{0}\right)^{-1}$. As a result, we obtain

$$
E^{0}=\left(\mathcal{G}_{S-1}^{0}\right)^{-1} \mathcal{P}_{S}^{0} .
$$

If $\left\|E^{0}\right\|=\sqrt{\left(v_{1}^{0}\right)^{2}+\cdots+\left(v_{m}^{0}\right)^{2}} \leq \varepsilon$, then $R^{0}=$ $\left(r_{1}^{0}, \ldots, r_{m}^{0}\right)^{T}$ is an $\varepsilon$-solution of the system (22).
Otherwise, according to (16), we take $R^{1}=$ $\left(\frac{r_{1}^{0}}{1-v_{1}^{0}}, \ldots, \frac{r_{m}^{0}}{1-v_{m}^{0}}\right)^{T}$ and we will move to the next iteration.

The iterative process continues until at the iteration with number $K$ we will have the condition

$$
\left\|E^{K}\right\|=\sqrt{\left(v_{1}^{K}\right)^{2}+\cdots+\left(v_{m}^{K}\right)^{2}}<\varepsilon,
$$

or until the number of iterations exceeds the allowable value be $K_{\max }$.

Let $I_{m \times m}$ is an identity matrix of $m \times m$ size, and $I_{m \times 1}=(1, \ldots, 1)$ is a column matrix of $m \times 1$ size with unit elements. Then, taking into account (16) and (22), identifying the $m$-dimensional vectors $R^{k}=\left(r_{1}^{k}, \ldots, r_{m}^{k}\right)^{T} \quad$ and $\quad E^{k}=\left(\mathcal{G}_{S-1}^{k}\right)^{-1} \mathcal{P}_{S}^{k}=$ $\left(v_{1}^{k}, \ldots, v_{m}^{k}\right)^{T}:\left\{k \in \mathbb{N}_{0}, k<K_{\max }\right\} \quad$ with $m \times 1$ column matrices, we obtain $R^{k+1}=I_{m \times m}\left(I_{m \times 1}-\right.$ $\left.E^{k}\right)^{-1} R^{k}$, or

$$
R^{k+1}=I_{m \times m}\left[I_{m \times 1}-\left(\mathcal{G}_{S-1}^{k}\right)^{-1} \mathcal{P}_{S}^{k}\right]^{-1} R^{k},
$$

It is easy to see that for $m=1$ formula (24) is transformed to form (10) and, therefore, can be assigned to the family of iterative formulas of the Laguerre's type.

Considering, that

$$
R^{k}=I_{m \times m}\left[I_{m \times 1}-\left(\mathcal{G}_{S-1}^{k-1}\right)^{-1} \mathcal{P}_{S}^{k-1}\right]^{-1} R^{k-1},
$$

we get

$$
\begin{gathered}
R^{k}=I_{m \times m}\left[I_{m \times 1}-\left(\mathcal{G}_{S-1}^{k-1}\right)^{-1} \mathcal{P}_{S}^{k-1}\right]^{-1} \cdot \ldots \\
I_{m \times m}\left[I_{m \times 1}-\left(\mathcal{G}_{S-1}^{0}\right)^{-1} \mathcal{P}_{S}^{0}\right]^{-1} R^{0},
\end{gathered}
$$

which implies that iterative process (24) is convergent if, for some $K: R^{K+1}=R^{K}$,

$$
\exists K \in \mathbb{N}: \lim _{k \rightarrow K}\left(\mathcal{G}_{S-1}^{k}\right)^{-1} \mathcal{P}_{S}^{k}=0 \text { or } \lim _{k \rightarrow K}\left\|E^{k}\right\|=0 .
$$

For $m=1$, this condition has the form corresponding to (10)

$$
x_{k}=\frac{x_{k-1}}{1-v_{k-1}}=\frac{x_{0}}{\left(1-v_{k-1}\right) \cdot \ldots \cdot\left(1-v_{0}\right)},
$$

and taking into account that $v_{k}=\frac{p_{n}\left(x_{k}\right)}{g_{n-1}\left(x_{k}\right)}$, we can assume that there is such number $k=K \in \mathbb{N}$, that $v_{K}=0$ and, therefore $p_{n}\left(x_{K}\right)=0$.

Let define a multi-metric in the metric space $\mathbb{R}^{m}$

$$
\operatorname{Dist}\left[F\left(R^{1}\right), F\left(R^{2}\right)\right] \leq L \cdot \operatorname{Dist}\left(R^{1}, R^{2}\right) .
$$

Schroeder's fixed point theorem [13].

Let the mapping $F: X \rightarrow \mathbb{R}^{m}$ is contracting on a closed subset $X$ of the space $\mathbb{R}^{m}$ with the multi- 
metric Dist. Then, for any $R^{0}$ the iterations sequence $R^{k+1}=F\left(R^{k}\right), k=0,1, \ldots$ converges to a single fixed point $R^{*}$ of the mapping $F$ in $X$ and takes place estimate

$$
\operatorname{Dist}\left(R^{k}, R^{*}\right) \leq(I-L)^{-1} L \cdot \operatorname{Dist}\left(R^{k}, R^{k-1}\right) .
$$

Thus, iterative process (24) will converge not for all $R^{0}$ (in the one-dimensional case $x_{0}$ ). To ensure convergence, it is necessary that the mapping (24) be compressing and that Schroeder's fixed point theorem be satisfied.

As an example, consider a system of two polynomial equations

$$
\left\{\begin{array}{l}
P_{1}\left(x_{1}, x_{2}\right)=4 x_{1}^{3}-3 x_{1}-x_{2}=0, \\
P_{1}\left(x_{1}, x_{2}\right)=x_{1}^{2}-x_{2}=0
\end{array}\right.
$$

with well-known real solutions:

$$
[0,0],[-0.75,0.5625],[1.0,1.0] \text {. }
$$

We describe in detail the iterative process (24), taking into account that the Jacobian of the system (26) $\mathbb{J}=12 x_{1}^{2}-2 x_{1}-3$ vanishes at $x_{1}=x_{J 1}=$ -0.4236 and $x_{2}=x_{J 2}=0.5902$.

For arbitrary $x_{1}$ and $x_{2}$, the system (26), taking into account (24), takes the following form:

$$
\begin{gathered}
\mathcal{H}(\mathcal{N}, X)=P_{S}(X)-G_{S-1}(X) \mathcal{N}= \\
\left(\begin{array}{c}
4 x_{1}^{3}-3 x_{1}-x_{2} \\
x_{1}^{2}-x_{2}
\end{array}\right)+\left(\begin{array}{cc}
6 x_{1} & -4 x_{1}^{2} \\
2 x_{2} & -x_{1}^{2}
\end{array}\right)\left(\begin{array}{l}
v_{1} \\
v_{2}
\end{array}\right)=\left(\begin{array}{l}
0 \\
0
\end{array}\right) .
\end{gathered}
$$

Solution of the system (27) is a vector

$$
\left(\begin{array}{l}
v_{1}\left(x_{1}, x_{2}\right) \\
v_{2}\left(x_{1}, x_{2}\right)
\end{array}\right)=\left(\begin{array}{c}
\frac{x_{2}\left(4 x_{1}^{2}-x_{1}-3\right)}{2 x_{1}^{2}\left(4 x_{2}-3\right)-3 x_{1}\left(2 x_{1}+x_{2}\right)} \\
\frac{2 x_{1}^{3}\left(4 x_{2}-3\right)+x_{2}\left(x_{2}-3 x_{1}^{2}\right)}{x^{1}\left[2 x_{1}^{2}\left(4 x_{2}-3\right)-3 x_{1}\left(2 x_{1}+x_{2}\right)\right]}
\end{array}\right) \text {. }
$$

Thus, the mapping (24), which for this example has the form $E]^{-1} R$,

$$
R=I_{2 \times 2}\left[I_{2 \times 1}-\mathcal{G}^{-1} \mathcal{P}\right]^{-1} R=I_{2 \times 2}\left[I_{2 \times 1}-\right.
$$

can be represented as

$$
\left(\begin{array}{l}
x_{1} \\
x_{2}
\end{array}\right)=\left(\begin{array}{c}
\frac{x_{1}\left[2 x_{1}^{2}\left(4 x_{2}-3\right)-3 x_{2}\left(x_{1}+2\right)\right]}{2 x_{1}^{2}\left(2 x_{2}-3\right)-x_{2}\left(2 x_{1}+3\right)} \\
\frac{x_{1}\left[2 x_{1}^{2}\left(4 x_{2}-3\right)-3 x_{2}\left(x_{1}+2\right)\right]}{6 x_{1}+x_{2}}
\end{array}\right) .
$$

It is easy to see that equations of the system (28) are identities in two cases: 1) $\left(\begin{array}{l}x_{1} \\ x_{2}\end{array}\right)=\left(\begin{array}{c}-0.75 \\ 0.5625\end{array}\right)$; 2) $\left(\begin{array}{l}x_{1} \\ x_{2}\end{array}\right)=\left(\begin{array}{l}1.0 \\ 1.0\end{array}\right)$, i.e., when $x_{1}$ and $x_{2}$ are nonzero solutions of system (26). In the case $\left(\begin{array}{l}x_{1} \\ x_{2}\end{array}\right)=\left(\begin{array}{l}0 \\ 0\end{array}\right)$, elements of the vector (28) take infinite values and, therefore, trivial solution of the system (26) cannot be obtained iteratively.

Now, to find $\varepsilon$-solutions of the system (26) using the iterative process (24), we set the following initial approximations: $x_{1}^{0}=x_{J 1}=-0.4236$ and $x_{2}^{0}=1.5$. That is, consider one of cases when, as an initial approximation, the value $x_{1}$ is selected, for which $\mathbb{J}=0$. The iterative process (24) in such case converges and at the 7 th iteration we obtain $\varepsilon$ solution $\left(\begin{array}{l}x_{1} \\ x_{2}\end{array}\right)=\left(\begin{array}{l}-0.75 \\ 0.5625\end{array}\right)$ of the system with the required accuracy. The dynamics of the iterative process for this case of initial data is shown in Fig. 1.
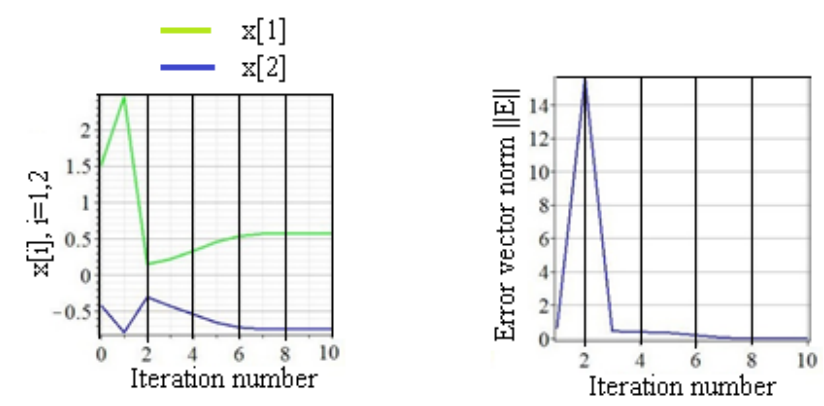

Fig.1. The dynamics of the iterative process (24) for solving the system (26) with initial approximations:

$$
\mathrm{x}_{1}^{0}=\mathrm{x}_{\mathrm{J} 1}=-0.4236 \text { and } \mathrm{x}_{2}^{0}=1.5 \text {. }
$$

That is, iterative processes implemented in accordance with the method presented above converge in cases where the Jacobi matrix of the system of equations is poorly conditioned or even degenerate. This is explained by the structure of formula (24), a special case of which for $m=1$ is the formula (10).

At the same time, iterative processes will obviously diverge when $\left\|I_{m \times 1}-\left(\mathcal{G}_{S-1}^{k}\right)^{-1} \mathcal{P}_{S}^{k}\right\| \approx$ 0 . This fact must be taken into account when designing software modules for solving polynomial equations systems of general form.

The homotopy of polynomial equations system, which in the case $m=2$ has the form (27), leads to the deformation of all equations with parameters $v_{1}, v_{2}$. In the above example, using (26), we obtain:

$$
\begin{gathered}
P_{\nu}^{1}(X)=4 x_{1}^{3}-3 x_{1}-x_{2}+6 x_{1} v_{1}-4 x_{1}^{2} v_{2}, \\
P_{v}^{2}(X)=x_{1}^{2}-x_{2}+2 x_{2} v_{1}-x_{1}^{2} v_{2} .
\end{gathered}
$$

If to use the terminology accepted above then polynomials (29) and (30) can be called multivariant locally convex polynomials that define the homotopy $\mathcal{H}(\mathcal{N}, X)$ of the system of two polynomial equations (26) with the vector of real parameters $\mathcal{N}=\left(\begin{array}{l}v_{1} \\ v_{2}\end{array}\right)$. Obviously, in the case when 
investigated the system of $m$ equations, the vector $\mathcal{N}$ will have the dimension $m$.

Fig. 2 shows graphs of multi-variant LRPfunctions $f_{v}^{1}(X)=P_{v}^{1}(X)$ and $f_{v}^{2}(X)=P_{v}^{2}(X)$, constructed for different values $v_{1}$ and $v_{2}$, which illustrate homotopy of the system (26).
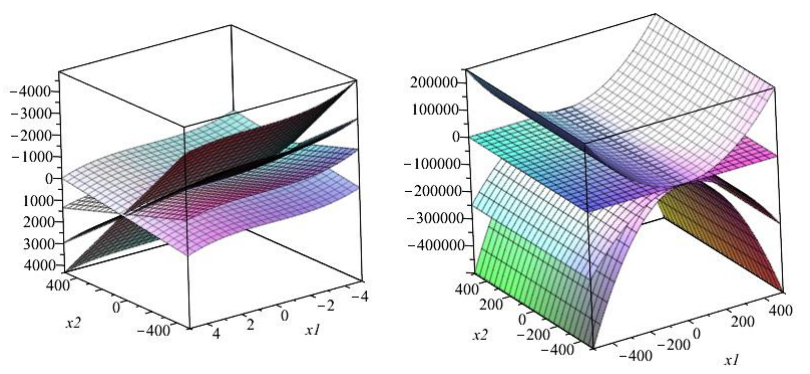

Fig.2. Graphs of multi-variant LRP-functions $\mathrm{f}_{v}^{\mathrm{i}}(\mathrm{X})=\mathrm{P}_{v}^{\mathrm{i}}(\mathrm{X}), i=1,2$, which illustrate homotopy of the system of polynomial equations (26) for various values of real parameters $v_{1}$ and $v_{2}: 1 . v_{1}=v_{2}=0$;

2. $v_{1}=1.0, v_{2}=2.0 ; 3 . v_{1}=2.0, v_{2}=1.0 ; 4$. $\left.v_{1}=3.0, v_{2}=3.0: a\right) \mathrm{f}_{v}^{1}(\mathrm{X})=\mathrm{P}_{v}^{1}(\mathrm{X})$; б) $\mathrm{f}_{v}^{2}(\mathrm{X})=$ $\mathrm{P}_{v}^{2}(\mathrm{X})$.

It is easy to see that all graphs of functions $f_{v}^{i}(X), i=1,2$ in Fig. 2 are distorted with respect to graphs of functions $f_{0}^{i}(X)$ as parameters values $v_{1}$ and $v_{2}$ are changed. Moreover, for any $v_{1}$ and $v_{2}$ they intersect along strictly defined lines. Obviously, that points at which such lines intersect, intrinsic to functions $f_{v}^{1}(X)$ and $f_{v}^{2}(X)$, respectively, are solutions of the system (27) for any values of real parameters $v_{1}$ and $v_{2}$, which determine only the degree of homotopy $\mathcal{H}(\mathcal{N}, X)$ with respect to $P_{S}(X)$. Moreover, if Schroeder theorem conditions for some initial approximations are satisfied then $\lim _{k \rightarrow \infty} v_{1} \rightarrow 0, \lim _{k \rightarrow \infty} v_{2} \rightarrow 0$ and $\mathcal{H}(\mathcal{N}, X) \rightarrow P_{S}(X)$.

\section{Numerical Examples}

We will give are some more examples illustrating practical implementation of the iterative process (24) in solving various nonlinear polynomial equations systems.

A. An almost linear Brown's system [14].

$$
\left\{\begin{array}{l}
2 x_{1}+x_{2}+x_{3}+x_{4}+x_{5}=6 \\
x_{1}+2 x_{2}+x_{3}+x_{4}+x_{5}=6 \\
x_{1}+x_{2}+2 x_{3}+x_{4}+x_{5}=6 \\
x_{1}+x_{2}+x_{3}+2 x_{4}+x_{5}=6 \\
x_{1} x_{2} x_{3} x_{4} x_{5}=1
\end{array}\right.
$$

As initial approximations were chosen sufficiently large numbers (in absolute value) for a given system: $\quad x_{1}^{0}=8.0, x_{2}^{0}=6.0, x_{3}^{0}=$ $4.0, x_{4}^{0}=1.0, x_{5}^{0}=-2.0$. The dynamics of the iterative process for solving system (31) is shown in Fig. 3.
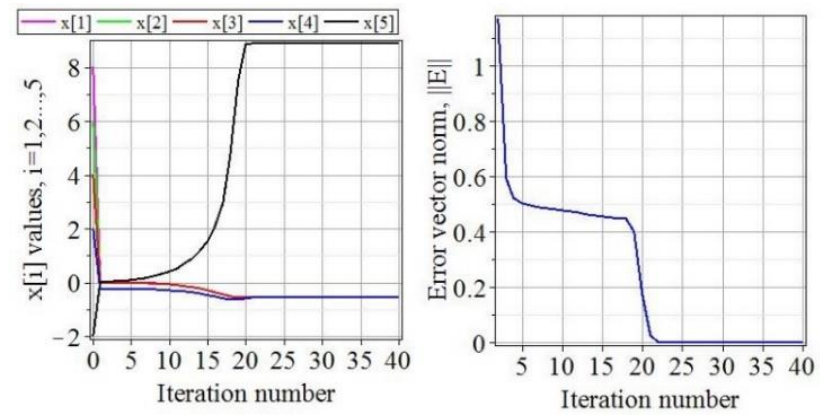

Fig.3. The dynamics of the iterative process (24) for solving system (31) with initial approximations: $x_{1}^{0}=8.0, x_{2}^{0}=6.0, x_{3}^{0}=4.0, x_{4}^{0}=1.0, x_{5}^{0}=-2.0$

It should be noted that in the considered example, to obtain the $\varepsilon$-solution with the required accuracy, a large number of iterations $\mathrm{K}=20$ were required. However, when choosing initial approximations in sufficiently small neighborhoods of the roots, the desired solutions were obtained with a much smaller number of iterations. [14].

B. The problem of the intersection of circles

$$
\left\{\begin{array}{l}
\left(x_{1}-a_{10}\right)^{2}+\left(x_{2}-a_{20}\right)^{2}=r^{2}, \\
\left(x_{1}-b_{10}\right)^{2}+\left(x_{2}-0.5\right)^{2}=\left(0.5-b_{10}\right)^{2},
\end{array}\right.
$$

where $\quad a_{20}=0.5+\left(a_{10}-0.5\right) \operatorname{tg} \vartheta ; \quad r=\frac{a_{10}-0.5}{\cos \vartheta}$; $\vartheta=1^{\prime} ; a_{10}=100 ; b_{10}=-100$.

As noted in [14], the process of finding solutions to the system (32) using the bisection method for given initial data is very difficult. Using formula (24) with initial approximations $x_{1}^{0}=0.01$ and $x_{2}^{0}=-0.8$, the iterative process turned out to converge and the desired solution $\left(\begin{array}{l}x_{1} \\ x_{2}\end{array}\right)=$ $\left(\begin{array}{c}0.4999576 \\ 0.52918069\end{array}\right)$ was obtained for $K=12$ iterations.

The dynamics of the iterative process 24 for the solving of the system (32) is shown in Fig. 4.

C. Combustion chemistry problem [14]

This is a real practical task of burning hydrocarbons in case of excess fuel. Its mathematical model can be represented by a system of four polynomial equations 


$$
\left\{\begin{array}{l}
P(X, A)=0 \\
P(X, B)=0 \\
x_{1}^{2}-x_{2}=0 \\
x_{4}^{2}-x_{3}=0
\end{array}\right.
$$

where $\quad P(X, A)=\alpha_{1} x_{2} x_{4}+\alpha_{2} x_{2}+\alpha_{3} x_{1} x_{4}+$ $\alpha_{4} x_{1}+\alpha_{5} x_{4} ; P(X, B)=\beta_{1} x_{2} x_{4}+\beta_{2} x_{1} x_{3}+$ $\beta_{3} x_{1} x_{4}+\beta_{4} x_{3} x_{4}+\beta_{5} x_{3}+\beta_{6} x_{4}+\beta_{7}$ $\alpha_{1}=-1.697 \cdot 10^{7} ; \alpha_{2}=2.177 \cdot 10^{7} ; \alpha_{3}=$ $0.55 ; \alpha_{4}=0.45 ; \beta_{1}=1.585 \cdot 10^{14} ; \beta_{2}=4.126$. $10^{7} ; \beta_{3}=-8.285 \cdot 10^{6} ; \beta_{4}=2.284 \cdot 10^{7} ; \beta_{5}=$ $-1.918 \cdot 10^{7} ; \beta_{6}=48.4 ; \beta_{7}=-27.73$.
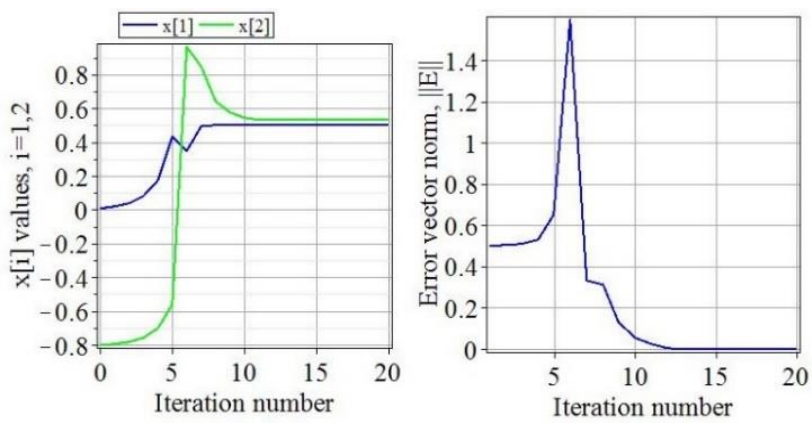

Fig.4. The dynamics of the iterative process (24) for the solving of the system (32) with initial approximations: $x_{1}^{0}=0.01$ and $x_{2}^{0}=-0.8$

As it is easy to notice, the main problems that may arise in the process of solving the system (33) are determined by the significant difference in the values of the coefficients and their absolute values. When using formula (24), these problems manifested themselves in the initial iterations (see Figure 5). Nevertheless, as a result of $K=28$ iterations, a unique solution was obtained in the domain of small values of variables:

$$
\left(\begin{array}{l}
x_{1} \\
x_{2} \\
x_{3} \\
x_{4}
\end{array}\right)=\left(\begin{array}{c}
-0.0001588472 \\
2.523242375 \cdot 10^{-8} \\
0.1478747693 \\
0.3845448859
\end{array}\right) \text {. }
$$

In this case, the following values were taken as initial approximations: $x_{1}^{0}=2.0, x_{2}^{0}=x_{3}^{0}=x_{4}^{0}=$ 0.01 .

The dynamics of the iterative process (24) for the solving of the system (33) is shown in Fig. 5.

\section{Conclusion}

In this paper, we analyze the capabilities of the iterative Laguerre's type method for numerically solving polynomial equations systems. It is shown that this method can be considered as one of the variants of the homotopy method, which allows one to efficiently find approximate solutions even in cases where the Jacobi matrix of the system is poorly conditioned, for example, with an unsuccessful choice of initial approximations.
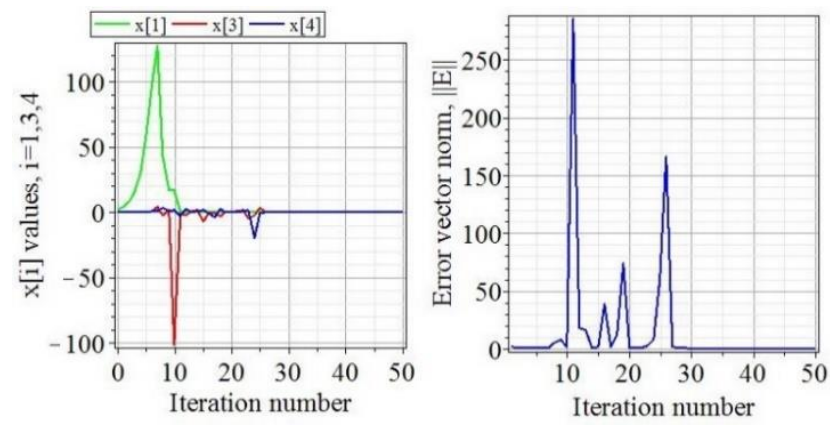

Fig.5. The dynamics of the iterative process (24) for solving system (33) with initial approximations:

$$
x_{1}^{0}=2.0, x_{2}^{0}=x_{3}^{0}=x_{4}^{0}=0.01 .
$$

Numerical experiments using systems of polynomial equations of various types have shown that in almost all cases this method is superior in efficiency to the classical Newton method. This is especially pronounced when the initial approximations are chosen, in general, arbitrarily. At the same time, in the case of choosing initial approximations in small neighborhoods of the desired solutions, the effectiveness of these two methods is almost the same.

Further research in this direction will be devoted to the development of the method with the aim of ensuring the possibility of its use for solving arbitrary nonlinear equations systems.

\section{Acknowledgments}

The reported study was funded of the internal grant 2020 by Sevastopol State University, project number 40/06-31.

\section{References:}

[1] S.N. Chow, J. Mallet-Paret, J.A. Yorke, Lecture Notes in Mathematics, in Functional differential equations and approximation of fixed points. Berlin: Springer Verlag, 1979.

[2] T.Y. Li, Foundations of Computational Mathematics, in Handbook of Numerical Analysis. Amsterdam:North-Holland, 2003.

[3] Y. Li and T. Sauer, Regularity results for solving systems of polynomials by homotopy method, Numerical Mathematics, Vol. 50, No. 3, 1987, pp. 283-289.

[4] T.Y. Li and X. Wang, Nonlinear homotopies for solving deficient polynomial systems with parameters, SIAM Journal on Numerical Analysis, Vol. 29, No. 4, 1992, pp. 1104-1118. 
[5] A.J. Sommese, J. Verschelde, C.W. Wampler, Homotopies for intersecting solution components of polynomial systems, SIAM Journal on Numerical Analysis, Vol. 42, No. 4, 2004, pp. 1552-1571.

[6] C.W. Wampler and A.P. Morgan, Solving the kinematics of general 6R manipulators using polynomial continuation, in Robotics: Applied Mathematics and Computational Aspects, Oxford: Clarendon Press, 1993.

[7] C.-H. Park and H.-T. Shim, What is the homotopy method for a system of nonlinear equations (survey), Journal of Applied Mathematics and Computing, Vol. 17, No. 1-23, 2005, pp. 689-700.

[8] O. Poliakov, M. Kolesova, Y. Pashkov, M. Kalinin, V. Kramar, Manipulator synthesis on the given properties of working space. Application of polynomials with linear real parameter, Proc. 2010 IEEE Int Conf on System, Man and Cybernetics, Istanbul, Turkye, 2010, pp. 4325-4332.

[9] A.M. Poliakov, Applications of a new numerical method for solving polynomial equations systems to problems of kinematics of hinge mechanisms, New Material and Technology in Metallurgy and Mechanical Engineerig, Vol. 1, 2012, pp. 120-126.

[10] E. Hansen and M. Patrick, A family of root finding methods, Numerical Mathematics, Vol. 27, 1977, pp. 257-269.

[11] O.N. Tikhonov, On the fast calculation of largest roots of a polynomial, Notes of Leningrad Institute name after G.V. Plekhanov, Vol. XLVIII, No. 3, 1968, pp. 36-41.

[12] A. Polyakov, A numerical solving algorithm for polynomial equation systems, Proc. of the 5th Triennial ETAI Int Conf on Applied Automatic Systems (AAS) 2009, Macedonia, Ohrid, 2009, pp. 109-112.

[13] J.M. Ortega and W.C. Rheinboldt, Iterative solution of nonlinear equation in several variables, N.-Y - London: Academic Press, 1970.

[14] R.P. Kearfott, Some tests of generalized bisection, ACM Transactions on Mathematical Software, Vol. 13, No. 3, 1987, pp. 197-220. 